\title{
K.B.M. - Mit einem interaktiven DaF-Theaterstück für Kinder auf Tournee durch Lateinamerika
}

\section{Alexander Riedmüller}

Ein Bericht über die außergewöhnliche Reise von Februar bis Juni 2013 des Wiener Theaterkolletivs artig durch Mittel- und Südamerika mit dem eigens dafür entwickelten Kinderstück „K.B.M. - Kleine bunte Männchen. " 2009 in Wien als studentische ImproTheatergruppe gegründet, arbeitet die Gruppe bestehend aus Magdalena Haftner, Lino Kleingarn, Anne-Marie Kuhfuß und Alexander Riedmüller heute international zusammen. Mittlerweile hat sie auf fünf Kontinenten, in über 100 Institutionen und auf mehreren Kongressen ihre Arbeit vorgestellt. Diese besteht aus Impro-Theaterauftritten, Workshops, Fortbildungen mit oder ohne DaF-Bezug und allem, was die vier reizt und nicht rasten lässt. K.B.M. ist eines dieser Projekte.

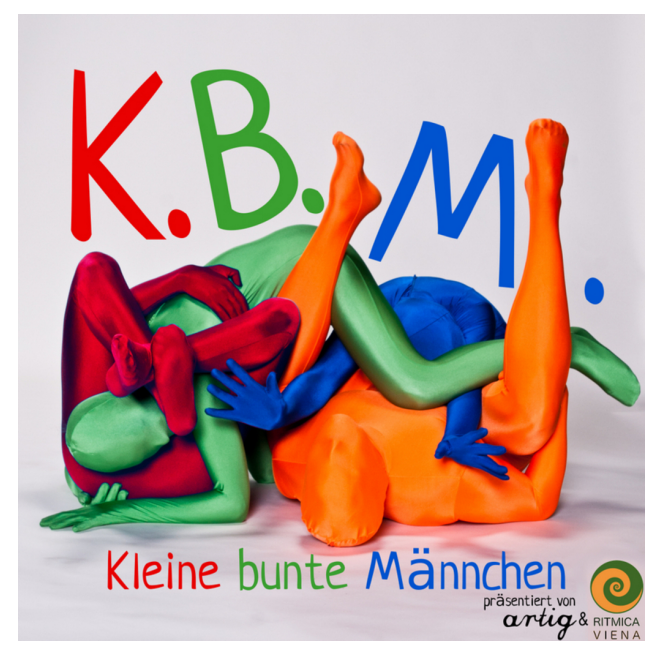

Der Naturwissenschaftsraum wird langsam immer voller. Zuvor waren hier noch nie 150 Kinder und ihre Lehrkräfte gemeinsam anwesend gewesen, aber heute sind alle Tische heraus geräumt und es sind viel mehr Sitzgelegenheiten da als gewöhnlich. Sämtliche Stühle, Bänke und davor eine Reihe Sitzkissen aus der Bibliothek und den angrenzenden Klassenzimmern sind auf die Ecke ausgerichtet, wo normalerweise die ausgestopfte Eule und der Bunsenbrenner neben dem Waschbecken stehen. Diese sieht man heute allerdings nicht, 
denn davor ist ein schwarzer Paravent aufgebaut. Dieses vor etwa 60 Minuten improvisiertes Versteck wurde aus Pinnwänden, Stehelementen und schwarzem Vorhang extra für diesen Anlass gebaut. Alles ist heute also besonders spannend für die Kinder der vierten Klassenstufe der Goethe-Schule in Buenos Aires. Doch auch hinter dem Vorhang ist die Stimmung gespannt. In etwa drei Minuten, dann wenn alle Kinder einen Platz gefunden haben, wird es losgehen. Kurz vorher werden auf diesen zwei Quadratmetern „Backstage-Bereich“ noch Klarinettenblätter befeuchtet, letzte Hautpartien mit blauer Farbe bedeckt, grüne Turnschläppchen zurecht gezogen und ein letzter Rest roter Farbe vom Mikrofon-Headset gewischt. Dass diese drei Farben nach vier Monaten Tournee fast sämtliche Kleidungsstücke der vier SchauspielerInnen Anne-Marie Kuhfuß, Lino Kleingarn, Magdalena Haftner und Alexander Riedmüller durchzogen haben, ist kein unwesentliches Detail dieser bewegten Reise durch sechs Länder und zu über 18 Schulen Lateinamerikas. Doch daran kann jetzt gerade niemand denken, denn es scheint so, als ob alle Kinder endlich einen Sitzplatz gefunden hätten. Ja, da kommt auch schon das abgemachte Signal im Versteck zum Vorschein, der Daumen der Deutschlehrerin nach oben, und das Team weiß, es kann losgehen.

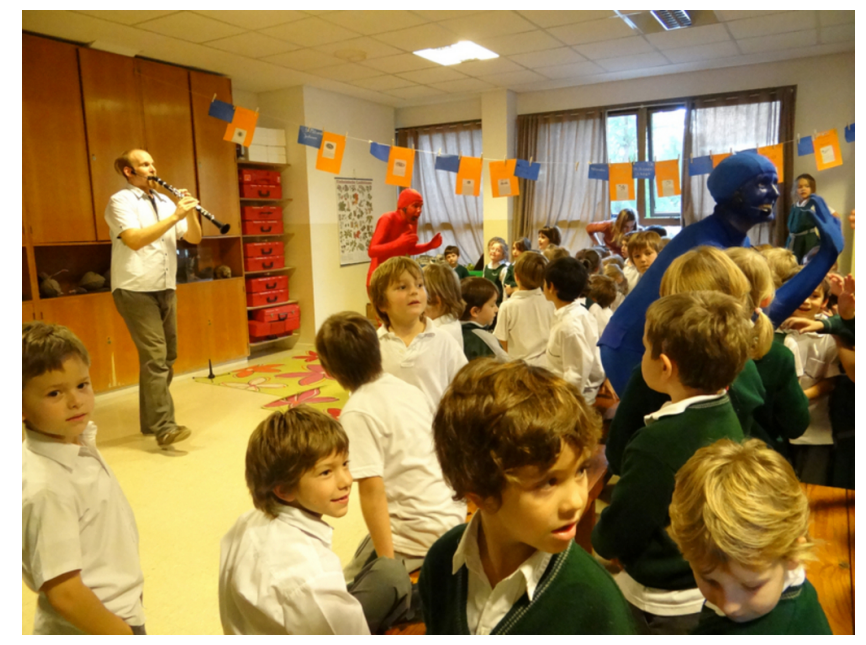

Einmal noch durchgeatmet und schon läuft Carlos wie von der Tarantel gestochen und mit seiner Klarinette in der Hand in den vollen Saal. Große Augen, Gekicher, auch einige etwas unsichere Gesichter, doch im Handumdrehen hat es der argentinische Musiker geschafft, durch seine Lieder und umgängliche Art eine Beziehung zu den Kindern aufzubauen. Er spricht nur Spanisch und wird von „furchtbaren bunten Wesen“ verfolgt, die er nicht verstehen kann. Carlos wundert sich, dass er auf seiner Flucht zufälligerweise schon wieder in einer deutschen Schule gelandet ist: „iOtra vez estoy en un Colegio Alemán!“ und erinnert sich an seine eigene herausfordernde und oft langweilige Schulzeit in einer solchen Institution. Er denkt jedoch auch an die schönen Momente seiner Kindheit, die er gemeinsam mit seiner „Oma“ und seinem „Opa“, die beide deutschsprachige Einwanderer waren, gehabt hat. Schnell ist dieser erste 
Moment der Ruhe aber vorbei, denn schon kommen sie, die K.B.M., die Kleinen bunten Männchen. Diese drei Kreaturen, die ausschließlich Deutsch sprechen und außerdem jeweils ein Lied, einen Tanz oder einen Rap nutzen, um sich vorzustellen, jagen Carlos und zunächst auch den Kindern einen gehörigen Schrecken ein. Während der nächsten 40 Minuten gewinnen sie allerdings nach und nach, auf ihre teils lustige und teils auch ziemlich coole Art und Weise, das Vertrauen der Kinder und schließlich sogar das des ängstlichen Carlos. Zum Glück können die SchülerInnen ja etwas Deutsch und übersetzen so einige Wörter für Carlos, der wortwörtlich nur „Bahnhof“ bzw. „Biene, Blume, Baum“ oder „Beethoven“ versteht. Am Ende dieses außergewöhnlichen Aufeinandertreffens tanzt er sogar gemeinsam mit den bunten Männchen, rappt auf Deutsch und freut sich riesig, dass er nun keine Angst mehr haben muss: Weder vor den fremden Wesen noch vor der deutschen Sprache.

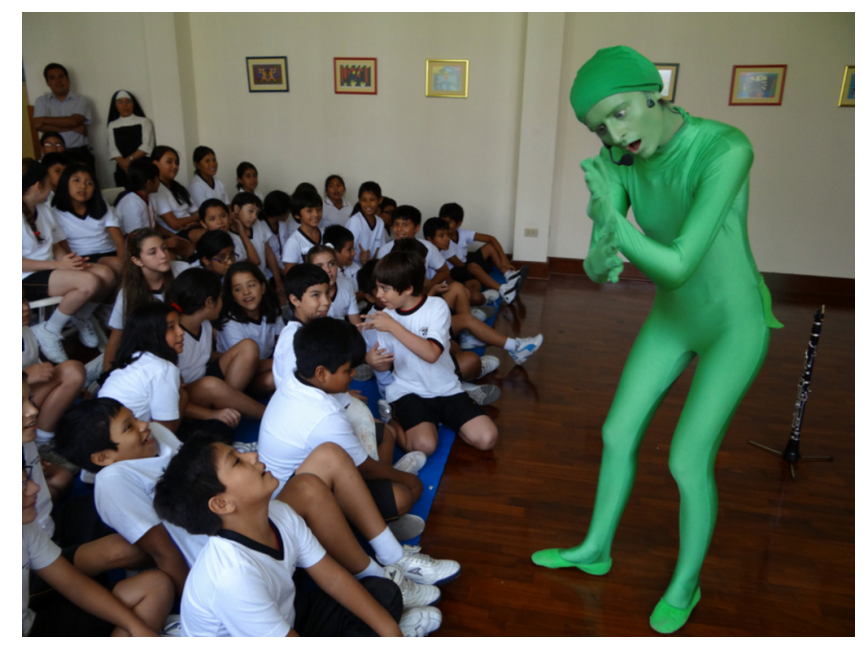

Den MacherInnen von KBM, den beiden Gruppen artig aus Wien und RITMICA VIENA aus Buenos Aires, war es besonders wichtig, mit diesem Stück die Angst vor Fremden zu thematisieren und gleichzeitig einen motivierenden Impuls für das Deutschlernen an Grundschulen in Lateinamerika zu geben. Einige dieser Schulen sind aus einer langen Tradition heraus deutsche Schulen. Das heißt, dass dort Deutsch einstmals als Muttersprache, jedoch mittlerweile meist als erste oder bereits zweite Fremdsprache gelehrt wird. Der muttersprachliche Anteil bei den vom KBM-Team besuchten Schulen lag bei nicht mehr als ein bis fünf Prozent. Da wollen gute Mittel gefunden werden, um die deutsche Sprache, die die Kinder im Alltag nicht oder kaum verwenden, zum attraktiven Lernobjekt zu machen. Dazu gehören in diesem Falle die interaktive Arbeitsweise unter Einbezug von vielen Liedern zum Mitsingen, Bewegungen zum Mitmachen, gezielte (Überraschungs-)Fragen an die Kinder sowie auch der unkonventionelle Aufführungsort, nämlich möglichst dort, wo normalerweise auch der Unterricht stattfindet, z.B. in einem Naturkunderaum mit ausgestopften Tieren und einer Fossiliensammlung.

Für die drei bunten Männchen und Carlos ist die Tournee mit dem 4. Juni 
2013 jedoch erst einmal vorbei und es kann gefeiert werden: Insgesamt sahen in fast 40 Aufführungen über 3500 Kinder dieses Stück und mehr als 600 Lehrkräfte wurden auf Deutsch, Spanisch und Portugiesisch zum Einsatz von Elementen der Theaterpädagogik und der Musik- und Bewegung (Rhythmik) fortgebildet. Fast ein Jahr dauerte die Vorbereitung. Alexander Riedmüller als Produzent koordinierte Aufführungen, Fortbildungen und Workshops teils im Rahmen des PASCH-Projekts des Goethe-Instituts und teils direkt mit den Schulen. Die Tournee endete dort, wo sie im Februar 2013 startete: in Buenos Aires. Danach ging das Stück, dass unter der Regie von Matías Nan gemeinsam mit den vier SchauspielerInnen von artig entwickelt wurde, auf Tour durch Kolumbien, Venezuela, Peru, Brasilien und Argentinien.

Für artig war dies nicht die erste Auslandsreise, denn seit 2011 ist das ja eigentlich in Wien ansässige Theaterkollektiv auf mehreren Kontinenten DaF-orientiert unterwegs. Sein Schwerpunkt liegt auf dem ImprovisationsTheater nach Keith Johnstone. Deshalb waren Haftner, Kuhfuß, Riedmüller und Kleingarn auch mit dem von ihnen entwickelten Konzept „spielend Deutsch“ unterwegs. Sie stellten das Regelwerk des Impro-Theaters in Kombination mit Sprachförderung auf dieser Tournee erstmals auch als DaF-Fortbildung oder Workshop an mehreren Schulen und Goethe-Instituten in Lateinamerika vor.

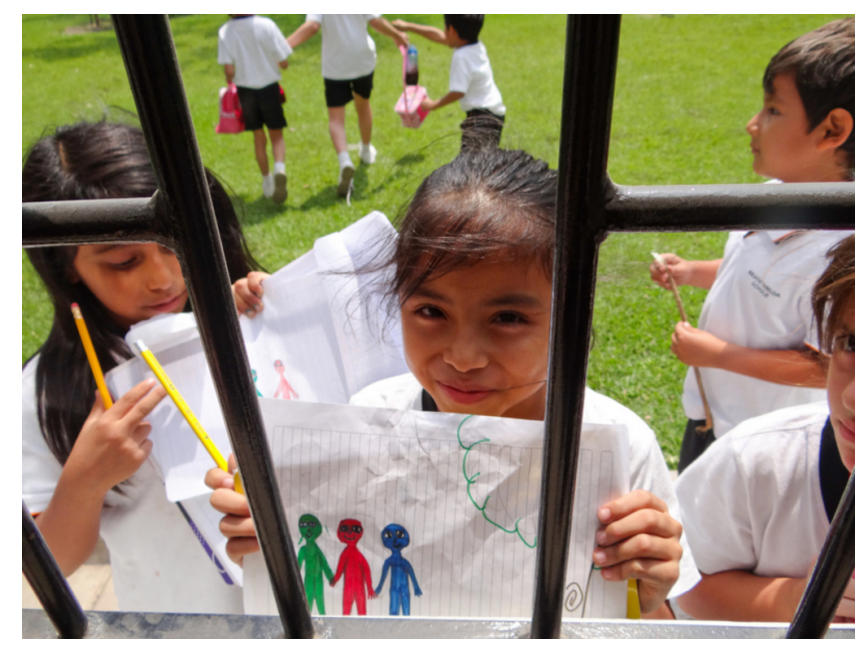

An diesem Tag, als die drei bunten Wesen und Carlos zum dritten Mal heute hinter dem Vorhang verschwinden, beendet die vierköpfige Gruppeihre intensive viermonatige Tournee. Es bleiben Erinnerungen an das Stück bei den Kindern, Lehrerinnen und Lehrern zurück und die vier SchauspielerInnen von artig zehren noch lange von den vielfältigen Eindrücken einer außergewöhnlichen und intensive Reise. 